\title{
F- Theory GUT's
}

\section{George K. Leontaris*}

Physics Department, Ioannina University, GR-45110 Ioannina, Greece E-mail: Leontaduoi.gr

In this lecture, an overview of current F-theory model building techniques is presented. In F-theory GUT symmetries are associated to the singularities of the elliptically fibred internal (compact) space. Models with gauge groups emerging as subgroups of the exceptional group $\mathscr{E}_{8}$ are presented. Moreover, GUTs with abelian and discrete symmetries arising from a non-trivial Mordell-Weil group associated to rational sections of the elliptic fibration are analysed.

Proceedings of the Corfu Summer Institute 2014 "School and Workshops on Elementary Particle Physics and Gravity",

3-21 September 2014

Corfu, Greece

\footnotetext{
* Speaker.
} 


\section{Introduction}

Phenomenological low energy models obtained from string theory are particularly interesting because many of their parameters are determined from a few basic principles of the theory at the high scale and a handful of topological properties of the compact space. F-theory [四] model building [[]]-[35] in particular is a promising component of the vast string landscape. Many properties of the effective F-theory models are determined from the elliptic structure of the fibred internal space. Furthermore, despite the complicated structure of the Calabi-Yau (CY) geometry, in the F-theory description one can focus on a small patch and compute several important quantities which can be directly confronted to experimental tests. For example, Yukawa couplings occur at triple intersections of D7-branes. Studying locally the wavefunctions' profiles of the relevant states we are able to compute the strength of these couplings and predict the mass spectrum and -at least in principleall possible interactions predicted within a specific model. Examples of such computations regarding vital issues such as proton decay, the Higgs mixing term and the fermion mass structure appear

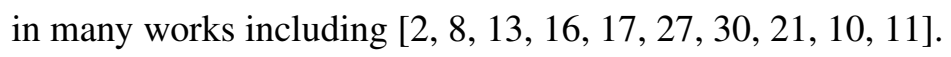

Another interesting fact in F-theory, is that GUT symmetries are linked to the singularities of the elliptically fibred $\mathrm{K} 3$ manifolds. The advantage in such constructions is that the exceptional groups $\mathscr{E}_{6,7,8}$, can be naturally incorporated into the theory (see for example [B], 团] and references therein). A systematic classification of semi-local F-theory GUTs arising from a single $\mathscr{E} 8$ point of local enhancement, leading to simple GUT groups based on $\mathscr{E}_{6}, S O(10)$ and $S U(5)$ have also been studied extensively.

In addition to the non-abelian sector, in string models, abelian and discrete symmetries are ubiquitous [36]-[45]. In F-theory models in particular these arise either as a subgroup of the nonabelian symmetry or from a non-trivial Mordell-Weil group associated to rational sections of the elliptic fibration. Phenomenological analysis shows that the role of abelian and discrete symmetries is particularly important in model building. They are the appropriate tool to suppress undesired proton decay operators and various flavour violating interactions and generate a hierarchical fermion mass spectrum.

In these lecture notes a short overview of the current status of F-theory model building is given, where the focus is mainly on the above issues. To make this presentation self-contained in the next two sections I review in brief the basics of elliptic curves, F-theory and elliptic fibration. The remaining sections are devoted to the methodology of current F-theory model building.

\section{Elliptic Curves}

F-theory [U] is a geometric reformulation of String Theory. Its 12-dimensional space consists of the four space-time dimensions and an eight-dimensional internal elliptically fibred compact space. Hence, the theory of elliptic curves plays a crucial role, while many of the properties that will be discussed are related to rational points on elliptic curves.

A point is said to be rational if its coordinates are rational, while a rational curve is defined by an equation with rational coefficients. It is trivial to find the rational points on lines and conics. Consider for example the equation of the unit circle $x^{2}+y^{2}=1$. Choosing a rational point on it, -let it be $(-1,0)$ - we can draw a line which intersects the circle at $(x, y)$. We can map this pair on 
the line identified with the vertical axis $y$ by the transformation

$$
x=\frac{1-t^{2}}{1+t^{2}}, y=\frac{2 t}{1+t^{2}},
$$

This way, all rational points $(x, y)$ on the curve can be determined in terms of the rational values of

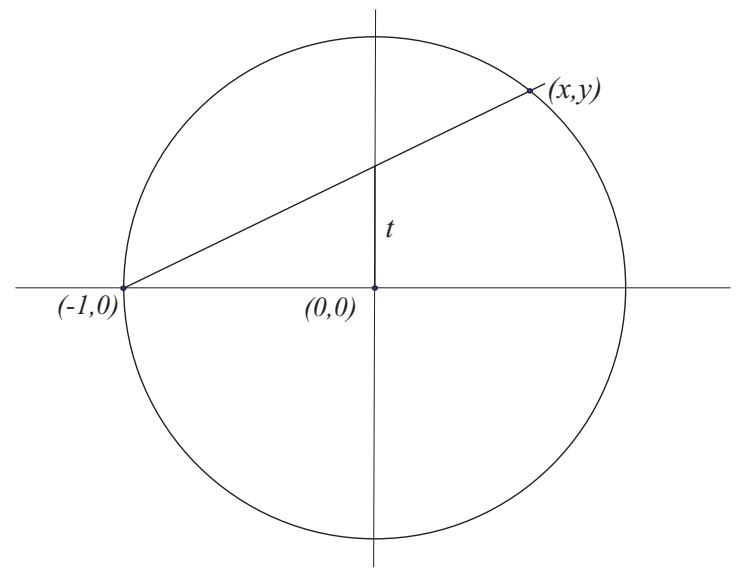

Figure 1: Rational points on conics.

the parameter $t$. Because of this correspondence between the rational points, we say that the curve (in this case the conic) is birationally equivalent to the line. A rational curve is also called a curve of genus zero. Every genus zero curve is birationally equivalent either to a conic or to a line.

We proceed now to the elliptic curves which are described by a cubic equation whose most general form can be written as

$$
\mathscr{C}: \sum_{n=0}^{3} \sum_{m=0}^{n} a_{m, n} x^{n} y^{n-m}=0
$$

The identification of the rational points on a given elliptic curve $\mathscr{C}$ of general type is much more complicated compared to the conic. We know however, that the rational points of $\mathscr{C}$ exhibit a group structure. According to Mordell's theorem,

If a non-singular elliptic curve $\mathscr{C}$ has a rational point then the group of rational points can be finitely generated.

In other words, there is a finite number of elements generating the whole group. The group structure is depicted here in figure $\square$ which can be defined as follows: Let $P, Q$ two rational points on $\mathscr{C}$. Drawing the line joining these two points, we can find another one at the third intersection of the line with the curve $\mathscr{C}$. I designate this point with $P * Q$. Suppose now we are given a rational point $O$ on $\mathscr{C}$ that we can identify this to be the zero element of the group. The line from $O$ to $P * Q$ intersects $\mathscr{C}$ on another point which, as can be proved ${ }^{1}$, under the group law is the point $P+Q$. To find the opposite element with respect to the addition law of the group, we draw a tangent to the zeroth element $O$ which intersects $\mathscr{C}$ at some point called here $S$. One can prove that the opposite to $P$ is identified with the third intersection of the $P S$-line and curve $\mathscr{C}$, so that $P+(-P)=O$.

\footnotetext{
${ }^{1}$ See for example standard textbooks such as [46, ,47, 48]].
} 

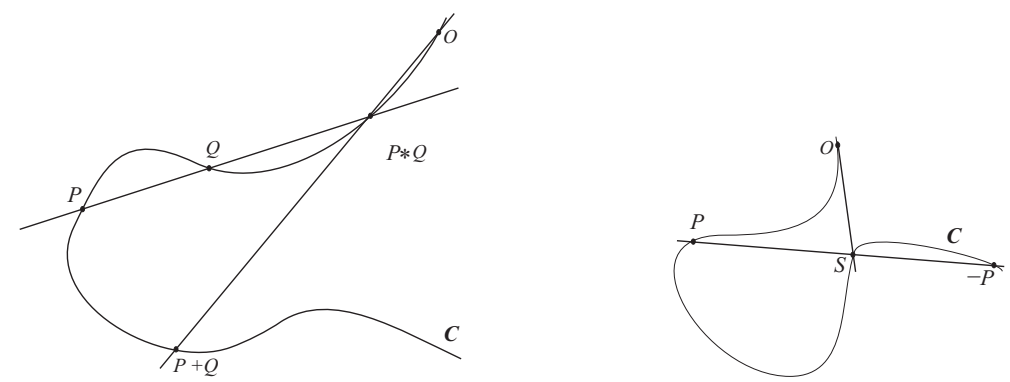

Figure 2: The group structure of rational points on elliptic curves. The law of addition.

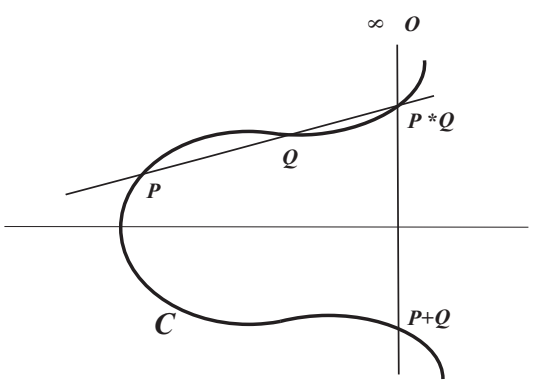

Figure 3: Addition on Weierstrass form. The zero element $\mathscr{O}$ is at infinity.

The general form (2.]) of the elliptic equation is rather too complicated. Fortunately it can be shown that any cubic equation with a rational point on it can be brought to the Weierstraß form

$$
y^{2}=x^{3}+f x+g
$$

We can readily check that the Weierstraß form is symmetric with respect to the $x$-axis. Moreover, the zeroth element of the group can be taken to infinity while the sum of two points is just the reflection (w.r.t. $x$-axis) of the third intersection point of the line PQ with $\mathscr{C}$.

There are two important quantities characterising the elliptic curves. These are:

- The discriminant

$$
\Delta=4 f^{3}+27 g^{2}
$$

which classifies the singularities on the elliptic curve. In particular, when $\Delta \neq 0$ elliptic curves are non-singular and may have one or three real roots. When $\Delta=0$ curves are singular. Singularities are of nodal or cuspidal type.

- The j-invariant (modular invariant) function

$$
j(\tau)=\frac{4(24 f)^{3}}{4 f^{3}+27 g^{2}}=\frac{4(24 f)^{3}}{\Delta}
$$

which takes the same value for equivalent elliptic curves characterised by the $S L(2, Z)$ transformations $\tau \rightarrow \frac{a \tau+b}{c \tau+d}$.

These two functions will be very useful when discussing the elliptically fibred space in Ftheory. 

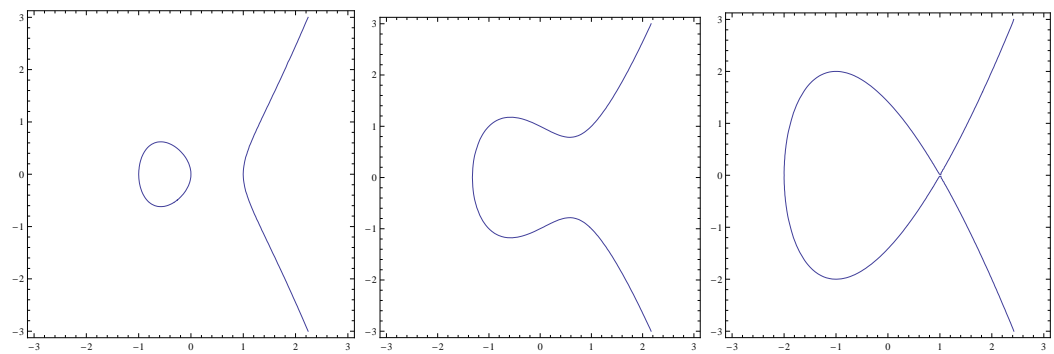

Figure 4: Fixing the values of the polynomials f,g to certain real numbers, elliptic fibrations reduce to elliptic curves. The three figures correspond to a discriminant bigger, less or equal to zero respectively.

Often, a crucial step towards understanding the structure of singular fibers is the operation known as resolution of singularities. The resolution changes the singular part of the fiber but leaves essentially unchanged the smooth part. We describe with a simple example this procedure. Let the singular curve

$$
y^{2}=x^{3}+x=x\left(x^{2}+1\right)
$$

We take homogeneous coordinates $u, v$ so that $v y=u x$, and define the affine parameter $t=u / v$, so that

$$
y=t\left(t^{2}-1\right) \text { and } x=t^{2}-1
$$

This way we have resolved the singularity by embedding the curve in a higher dimensional space. (blowing-up a singularity). This is depicted in figure $[$.

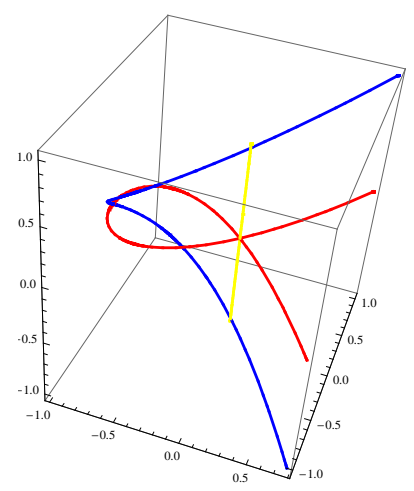

Figure 5: Blowing-up a singularity

So far we dealt with real elliptic curves, however our final goal is to use their complex analogues. Hence, we need to consider complex coefficients (functions) $f, g \in \mathbf{C}$. In this case, it can be proved that a complex elliptic curve $\mathscr{C}$ is a genus 1 closed surface with a marked point on it corresponding to its neutral element (point to infinity). Defining a modulus $\tau$ as usually, the torus (hence $\mathscr{C}$ ), is equivalent to the lattice $(1, \tau)$.

With respect to the previous analysis of elliptic curves, we distinguish two cases: The complex analogue of a real elliptic curve with non-singular points is a torus without singularities. On the contrary, if the real elliptic curve has singular points then its complex equivalent is a torus with a pinched radius. 


\section{Real Complex}

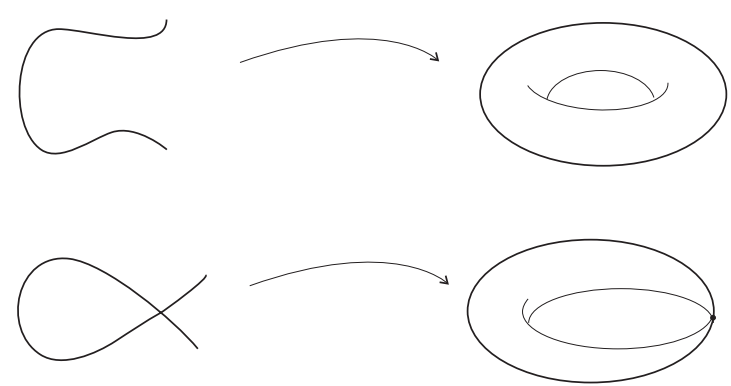

Figure 6: The Weierstraß equation with complex coefficients describes a torus. A non-singular real elliptic curve "upgrades" to normal torus. Singular elliptic curve corresponds to torus with a pinched radius.

\section{F-theory and Elliptic Fibration}

We start with the description of a few basic features of F-theory [四]. F-theory is defined on a background $R^{3,1} \times X$ with $R^{3,1}$ the 4-d space-time and $X$ an elliptically fibred CY complex fourfold with a section over a complex three-fold base $B_{3}$. In short, it is the geometrisation of the type II-B superstring.

There are several reasons for preferring to obtain a geometric picture of string theory. We will see that in the fibred internal space a significant role is played by the geometric singularities which are mathematically well understood and systematically classified. F-theory is based on the idea that geometry dictates the group structure of the effective field theory model.

Let us start then with the type II-B superstring. In the bosonic spectrum there are two scalars, the dilaton field $\phi$ and a zero-form potential (axion) $C_{0}$. One then can define a modulus, the axiondilaton complex structure

$$
\tau=C_{0}+i e^{-\phi}
$$

and write down an $S L(2, Z)$ invariant action of the ten-dimensional theory which leads to consistent equations of motion. The terms of the action seem as if they are obtained from a twelve dimensional theory compactified along the two radii of the torus (for a review see [49]]). We can think of $\tau$ as the modulus of a torus attached to each point of the internal manifold of three complex dimensions (threefold), as depicted in figure $\square$. We end up with a fibred fourfold. Recalling the analysis of the previous section, one is tempted to consider the interesting possibility of describing this fibration by the Weierstraß model given in equation (2.2). We will approach this through a specific example establishing an equivalence between F-theory compactifications on a $\mathrm{K} 3$ surface and the heterotic theory compactification on $T^{2}$ [5] ].

\subsection{Elliptic K3 fibration}

A K3 surface is a complex smooth regular manifold with trivial canonical bundle. The general elliptically fibred K3 is described by the Weierstrass equation

$$
y^{2}=x^{3}+f(u, w) x z^{4}+g(u, w) z^{6}
$$




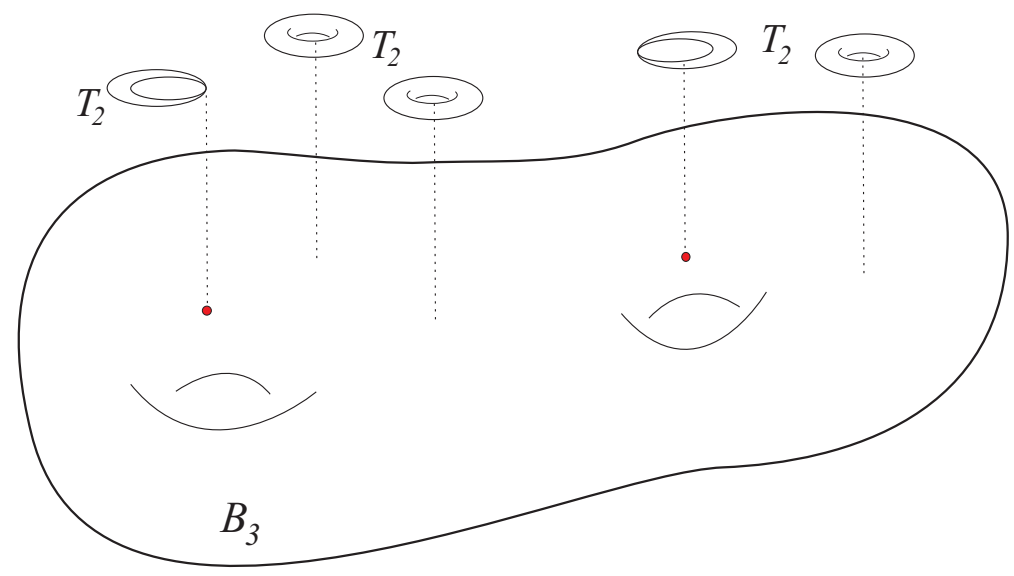

Figure 7: Elliptic fibration. At each point of the threefold $B_{3}$ a torus $\tau=C_{0}+i e^{-\phi}$ is assigned.

where $x, y, z, u, w$ are parameters of the fibration and $f, g$ homogeneous polynomials of degree 8 and 12 respectively. The equation is invariant under the following two rescalings

$$
\{u, w, x, y, z\} \rightarrow\left\{\lambda u, \lambda w, \lambda^{4} x, \lambda^{6} y, z\right\} ;\{u, w, x, y, z\} \rightarrow\left\{u, w, \mu^{2} x, \mu^{3} y, \mu z\right\}
$$

Indeed, for the first rescaling the left hand side becomes $y^{2} \rightarrow \lambda^{12} y^{2}$ and the same weight $\lambda^{12}$ emerges for the right hand side of (B.2). Similarly, one finds that for the second rescaling from both terms of the equation a weight $\mu^{6}$ is factored out.

There are five coordinates compared to two rescalings and one equation, thus the equation describes a two complex dimensional surface.

For the first rescaling we observe that the sum of the weights (powers of $\lambda$ ) is $1+1+4+6+$ $0=12$, i.e. equal to the weight 12 , and the second is $0+0+2+3+1=6$ is equal to the weight of the second equivalent equation. Therefore, this is a CY manifold.

Fixing $u=1$ the above equation becomes

$$
y^{2}=x^{3}+f(w) x z^{4}+g(w) z^{6}
$$

We now observe that $f, g$ transform as sections $f \in K_{B_{3}}^{-4}, g \in K_{B_{3}}^{-6}$. This can be understood if we assign the scalings $x \rightarrow \lambda^{2} x$ and $y \rightarrow \lambda^{3} y$ while taking $z=1$ so that (3.3) becomes $\lambda^{6} y^{2}=$ $\lambda^{6} x^{3}+\tilde{f} \lambda^{2} x+\tilde{g}$ implying $\tilde{f} \rightarrow \lambda^{4} f$ and $\tilde{g} \rightarrow \lambda^{6} g$.

For $z=1$ equation ([3.3) is just equation ([2.2). As explained previously, for $f, g$ complex, the latter describes a torus whose modulus $\tau$ is now identified with that of (B.Cl). On the other hand, in order to satisfy the CY conditions, we take the two functions $f=f(w), g=g(w)$ to be $8^{\text {th }}$ and $12^{\text {th }}$ degree polynomials of the complex variable $w$. As we move from point to point in the internal manifold, the modulus $\tau$ varies. In particular, on moving along non-trivial closed cycles, $\tau$ undergoes non-trivial $S L(2, Z)$ transformations ${ }^{2}$. In figure $\square$ for any generic point we draw a normal torus, while pinched torii are drawn at points of singularities; the latter appear when two D7-branes intersect at a 'point' of the manifold. These correspond to singularities of elliptic surfaces and

\footnotetext{
${ }^{2}$ The $S L(2, Z)$ modular invariant function is given by $j(\tau)=e^{-2 \pi i \tau}+744+\mathscr{O}\left(e^{-2 \pi i \tau}\right)$. Combined with ([2. $)$ one can elaborate [5] ] a relation approximated with $\tau(w) \sim \frac{1}{2 \pi i} \ln \left(w-w_{i}\right)$ in the vicinity of the zeros of $\Delta\left(w_{i}\right)=0$.
} 


\begin{tabular}{|l|l|l|l|l|}
$\operatorname{ord}(f)$ & $\operatorname{ord}(g)$ & $\operatorname{ord}(\Delta)$ & fiber type & Singularity \\
\hline 0 & 0 & $n$ & $I_{n}$ & $A_{n-1}$ \\
$\geq 1$ & 1 & 2 & $I I$ & none \\
1 & $\geq 2$ & 3 & $I I I$ & $A_{1}$ \\
$\geq 2$ & 2 & 4 & $I V$ & $A_{2}$ \\
2 & $\geq 3$ & $n+6$ & $I_{n}^{*}$ & $D_{n+4}$ \\
$\geq 2$ & 3 & $n+6$ & $I_{n}^{*}$ & $D_{n+4}$ \\
$\geq 3$ & 4 & 8 & $I V^{*}$ & $E_{6}$ \\
3 & $\geq 5$ & 9 & $I I I^{*}$ & $E_{7}$ \\
$\geq 4$ & 5 & 10 & $I I^{*}$ & $E_{8}$ \\
\hline
\end{tabular}

Table 1: The Kodaira classification relies on the vanishing order of the polynomials $f, g$ and the discriminant $\Delta$.

were classified in terms of the vanishing orders of the discriminant and the polynomials $f(w), g(w)$ several decades ago by Kodaira [ $[\mathbf{]}]$. For minimal elliptic surfaces eight types of singular fibers were identified, (nodal, cuspidal or otherwise reducible). The singularities are related to simplylaced Dynkin diagrams of ADE type. These extremely interesting results can be found in several recent papers and reviews [5], 52, 53], 54] . Here they are summarised in Table [.].

\section{Interpretation of singularities}

The nature of the singularities of the internal space motivated the idea that they can be identified with the gauge symmetries of the effective field theory model. If this is true, then one can attribute all the properties of the internal manifold to the massless spectrum and the effective potential describing their interactions. This scenario has many advantages, including calculability of Yukawa couplings [ [2]-[] [ $[2]$ of the effective theory from a handful of geometric characteristics of the internal space.

A convenient description which emphasizes the local properties of these singularities is given in terms of Tate's algorithm [55]. In this context, the equation describing the elliptically fibred space takes the form

$$
y^{2}+\alpha_{1} x y z+\alpha_{3} y z^{3}=x^{3}+\alpha_{2} x^{2} z^{2}+\alpha_{4} x z^{4}+\alpha_{6} z^{6}
$$

The variables $[x, y, z]$ have weights $[2: 3: 1]$ correspondingly, defining a hypersurface in the $\mathbf{P}_{(2,3,1)}$ weighted projective space.

In analogy with Kodaira's classification of singularities, here also the gauge group is determined in terms of the vanishing orders of the polynomials $\alpha_{k}$ and the discriminant $\Delta$. The results are summarised in Table $\square$. We note that the Weierstraß equation can be obtained from Tate's form by recovering the functions $f, g$ from the coefficients $\alpha_{k}$. To this end, it is convenient to define the following quantities

$$
\beta_{2}=\alpha_{1}^{2}+4 \alpha_{2} ; \beta_{4}=\alpha_{1} \alpha_{3}+2 \alpha_{4} ; \beta_{6}=\alpha_{3}^{2}+4 \alpha_{6} ; \beta_{8}=\frac{1}{4}\left(\beta_{2} \beta_{6}-\beta_{4}^{2}\right) .
$$




\begin{tabular}{|c|c|c|c|c|c|c|c|}
\hline Type & Group & $a_{1}$ & $a_{2}$ & $a_{3}$ & $a_{4}$ & $a_{6}$ & $\Delta$ \\
\hline$I_{0}$ & - & 0 & 0 & 0 & 0 & 0 & 0 \\
\hline$I_{1}$ & - & 0 & 0 & 1 & 1 & 1 & 1 \\
\hline$I_{2}$ & $S U(2)$ & 0 & 0 & 1 & 1 & 2 & 2 \\
\hline$I_{2 m}^{n s}$ & $S p(m)$ & 0 & 0 & $m$ & $m$ & $2 m$ & $2 m$ \\
\hline$I_{2 m}^{s}$ & $S U(2 m)$ & 0 & 1 & $m$ & $m$ & $2 m$ & $2 m$ \\
\hline$I_{2 m+1}^{s}$ & $S U(2 m+1)$ & 0 & 1 & $m$ & $m+1$ & $2 m+1$ & $2 m+1$ \\
\hline$I_{1}^{* s}$ & $S O(10)$ & 1 & 1 & 2 & 3 & 5 & 7 \\
\hline$I_{2 m-3}^{* n s}$ & $S O(4 m+1)$ & 1 & 1 & $m$ & $m+1$ & $2 m$ & $2 m+3$ \\
\hline$I_{2 m-3}^{* s}$ & $S O(4 m+2)$ & 1 & 1 & $m$ & $m+1$ & $2 m+1$ & $2 m+3$ \\
\hline$I_{2 m-2}^{* n n}$ & $S O(4 m+3)$ & 1 & 1 & $m+1$ & $m+1$ & $2 m+1$ & $2 m+4$ \\
\hline$I_{2 m-2}^{* n}$ & $S O(4 m+4)^{*}$ & 1 & 1 & $m+1$ & $m+1$ & $2 m+1$ & $2 m+4$ \\
\hline$I V^{* S}$ & $\mathscr{E}_{6}$ & 1 & 2 & 2 & 3 & 5 & 8 \\
\hline$I I I^{* s}$ & $\mathscr{E}_{7}$ & 1 & 2 & 3 & 3 & 5 & 9 \\
\hline$I I^{s}$ & $\mathscr{E}_{8}$ & 1 & 2 & 3 & 4 & 5 & 10 \\
\hline
\end{tabular}

Table 2: Selected cases of Tate's algorithm. The first column declares the type of the singular fiber according to Kodaira, i.e. nodal $\left(I_{1}\right)$, cuspidal $(I I)$ etc. The superscripts $s, n s$ stand for split and non-split. (The complete results can be found in [55, [52, 54]].) The other columns show the order of vanishing of the coefficients $a_{i} \sim z^{n_{i}}$, the discriminant $\Delta$ and the corresponding gauge group.

Then, it can be readily checked that the functions $f, g$ and the discriminant $\Delta$ are

$$
\begin{aligned}
f & =\frac{1}{48}\left(24 \beta_{4}-\beta_{2}^{2}\right) \\
g & =\frac{1}{864}\left(\beta_{2}^{3}-36 \beta_{4} \beta_{2}+216 \beta_{6}\right) \\
\Delta & =-8 \beta_{4}^{3}+9 \beta_{2} \beta_{6} \beta_{4}-27 \beta_{6}^{2}-\beta_{2}^{2} \beta_{8}
\end{aligned}
$$

After these preliminary notes, in the next section we proceed to the description of the basic tools for local model building.

\section{GUT models}

The attractive scenario of linking gauge symmetries to the singularities of the internal geometry leads to far reaching implications. An interesting advantage of F-theory constructions based on the elliptic fibration, is the appearance of the exceptional symmetry $\mathscr{E}_{8}$ where the gauge group

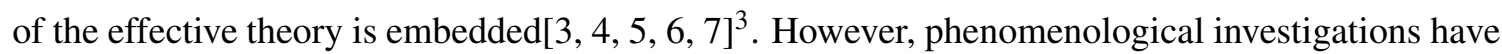
shown that additional symmetries (discrete or continuous) are required to render the theory viable. Interestingly, a useful class of such symmetries originates from the commutant of this GUT with respect to the exceptional gauge symmetry $\mathscr{E}_{8}$.

\footnotetext{
${ }^{3}$ For reviews, see [23], 241, 25], 26]
} 
To show how these symmetries appear we describe the $\mathscr{E}_{6}$ and $S U(5)$ gauge groups in brief. In the local picture, Tate's coefficients have a general expansion of the form

$$
\alpha_{k}=\alpha_{k 0}+\alpha_{k 1} w+\alpha_{k 2} w^{2}+\ldots
$$

If a certain coefficient $\alpha_{k}$ has vanishing order $n$, it is convenient to write

$$
\alpha_{k}=\alpha_{k, n} w^{n}, \text { with } \alpha_{k, n}=\alpha_{k n}+\alpha_{k(n+1)} w+\cdots
$$

We proceed to a brief description of the two aforementioned GUTs.

\section{$5.1 \mathscr{E}_{6}$ GUT}

For an $\mathscr{E}_{6}$ type of singularity, the coefficients take the form

$$
\alpha_{1}=\alpha_{1,1} w, \alpha_{2}=\alpha_{2,2} w^{2}, \alpha_{3}=\alpha_{3,2} w^{2}, \alpha_{4}=\alpha_{4,3} w^{3}, \alpha_{6}=\alpha_{6,5} w^{5}
$$

With this choice, the discriminant is factorised as follows

$$
\Delta=\Delta_{0} w^{8}
$$

where we defined

$$
\Delta_{0}=-27 \alpha_{3,2}^{4}+A\left(\alpha_{k j}\right) w+\mathscr{O}\left(w^{2}\right)
$$

while for convenience we introduced the coefficient $A\left(\alpha_{k j}\right)$ of the first power in $w$ :

$$
A\left(\alpha_{k j}\right)=\left(\alpha_{1,1} \alpha_{3,2}+2 \alpha_{4,3}\right)\left(\left(\alpha_{1,1}^{2}+36 \alpha_{2,2}\right) \alpha_{3,2}^{2}-32 \alpha_{4,3}\left(\alpha_{1,1} \alpha_{3,2}+\alpha_{4,3}\right)\right)-216 \alpha_{3,2}^{2} \alpha_{6,5}
$$

Indeed, $\Delta$ has a vanishing order of $8^{\text {th }}$ degree, in accordance with Table $\square$. From (5.3) we observe that the discriminant locus consists of two divisors, $D_{\mathscr{E}_{6}}$ (at $w=0$ of multiplicity eight) and $D_{\mathscr{I}}$ (at $\Delta_{0}=0$ of multiplicity one). There are eight $D 7$ branes wrapping the divisor $D_{\mathscr{E}_{6}}$ and one $D 7$ brane wrapping $\Delta_{\mathscr{I}}$ which is assumed to be irreducible.

The representations of the effective theory model, reside at the intersections of the $D_{\mathscr{E}_{6}}$ divisor with $\mathrm{D} 7$ branes spanning different dimensions of the internal space. These intersections (often called matter curves) are in fact Riemann surfaces along which symmetry is enhanced. In the elliptic fibration the highest allowed singularity is $\mathscr{E} 8$. Then, a convenient way to attain a viable effective model is through the decomposition

$$
\mathscr{E}_{8} \rightarrow \mathscr{E}_{6} \times S U(3)
$$

where $\mathscr{E}_{6}$ is the desired GUT, while the enhancements along the matter curves include factors embedded in $S U(3)$. We can also think of $S U(3)$ broken by fluxes (or some other mechanism) to a subgroup of it. The possibilities are either the continuous symmetries $S U(2), U(1)^{2}$, or a discrete group such as the $S_{3}$ (permutation of three objects), $Z_{3}$ or $Z_{2}$. Hence all $\mathscr{E}_{6}$ representations transform non-trivially under the latter. Viable cases have a final symmetry such as:

$$
\mathscr{E}_{6} \times U(1)^{2}, \mathscr{E}_{6} \times S_{3}, \mathscr{E}_{6} \times Z_{2}
$$


In general, for an $\mathscr{E}_{6}$ GUT, any additional symmetry embedded in $\mathscr{E}_{8}$ is included into its commutant, $S U(3)$. A phenomenologically simple description of these symmetries is based on the spectral cover approach and can be realised as follows. According to the previous discussion, the Tate's form for $\mathscr{E}_{6}$ is written

$$
y^{2}+\alpha_{11} x y w+\alpha_{32} y w^{2}=x^{3}+\alpha_{22} x^{2} w^{2}+\alpha_{43} x w^{3}+\alpha_{65} w^{5}
$$

Let us assign the following weights on the complex coordinates: $[x: y: w]=\left[\frac{1}{3}: \frac{1}{2}: 1\right]$. Dropping the terms with weights bigger than one, namely $[x y w]=\frac{31}{30}>1,\left[x^{2} w^{2}\right]=\frac{16}{10}>1$, we end up with

$$
y^{2}+\alpha_{32} y w^{2}=x^{3}+\alpha_{43} x w^{3}+\alpha_{65} w^{5}
$$

Defining a new parameter $s^{3}=w^{5}$ with $x=s^{-4 / 5}, y=s^{-6 / 5}$, we get

$$
\mathscr{C}_{3}: b_{0} s^{3}+b_{2} s+b_{3}=0
$$

where $b_{0}=\alpha_{65}, b_{2}=\alpha_{43}, b_{3}=-\alpha_{32}$. The three roots $t_{i}$ of the polynomial $\mathscr{C}_{3}$ are associated to the $S U$ (3) Cartan subalgebra satisfying $\sum_{i} t_{i}=0$. The coefficients $b_{k}$ encode the information of the compact space, while they can be written in terms of elementary functions of $t_{i}$. Inversion of these relations may lead to branchcuts, connecting some $t_{i}$ through monodromies. As a result, the original $S U$ (3) symmetry reduces to some discrete or continuous subgroup such as in (5.5). Details on viable models can be found in several papers including [ [6, B], B], B2]].

\section{$5.2 S U(5)$ GUT}

As a second example we consider that the GUT gauge symmetry is associated to a divisor characterised by an $S U(5)$ singularity while the commutant is also $S U(5)$-usually denoted with $S U(5)_{\perp}$.

$$
E_{8} \supset S U(5) \times S U(5)_{\perp}
$$

The implications of $S U(5)_{\perp}$ are described by a spectral cover denoted by $\mathscr{C}_{5}$ as follows: The coefficients fulfilling the conditions for the $S U(5)$ singularity are denoted as

$$
b_{0}=\alpha_{6,5}, b_{2}=\alpha_{4,3}, b_{3}=\alpha_{3,2}, b_{4}=\alpha_{2,1}, b_{5}=\alpha_{1,0}
$$

Define the homogeneous coordinates

$$
w \rightarrow U, x \rightarrow V^{2}, y \rightarrow V^{3}
$$

so that the Weierstrass equation becomes

$$
0=b_{0} U^{5}+b_{2} V^{2} U^{3}+b_{3} V^{3} U^{2}+b_{4} V^{4} U+b_{5} V^{5}
$$

with $U, V$ being sections of $-t$ and $c_{1}-t$ respectively ${ }^{4}$.

\footnotetext{
${ }^{4}$ As previously, $c_{1}$ the $1{ }^{\text {st }}$ Chern class of the tangent to $S_{G U T}$ bundle and $-t$ that of the normal bundle.
} 
We can write this equation in terms of the affine parameter $s=U / V$

$$
\mathscr{C}_{5}: \quad \sum_{k=0}^{5} b_{k} s^{5-k}=b_{0} s^{5}+b_{1} s^{4}+b_{2} s^{3}+b_{3} s^{2}+b_{4} s+b_{5}=0
$$

which is a five degree polynomial (notice that $b_{1}=0$ as expected for any $S U(n)$ group). Equation (5.7) includes the basic information regarding geometric properties as well as additional symmetries of the $S U(5)$ F-GUT.

The massless spectrum is found in $\mathbf{2 4 8}$ of $E_{8}$, which decomposes as follows

$$
248 \rightarrow(24,1)+(1,24)+(10,5)+(\overline{5}, 10)+(\overline{5}, \overline{10})+(5, \overline{10})
$$

The $S U(5)$ GUT multiplets have transformation properties under the second $S U(5)_{\perp}$. Depending on the specific topological structure of the internal space, the spectral cover $\mathscr{C}_{5}$ may factorise in various ways. A few interesting cases are

$$
\mathscr{C}_{4} \times \mathscr{C}_{1}, \mathscr{C}_{3} \times \mathscr{C}_{2}, \mathscr{C}_{2} \times \mathscr{C}_{2} \times \mathscr{C}_{1}
$$

implying analogous factorisations of the polynomial (5.7). For the $S U(5)$ GUT, there is a rich variety of possible accompanying discrete symmetries, including [27, [35, 144]

$$
S U(5) \times A_{4} \times U(1), S U(5) \times Z_{3} \times Z_{2}, S U(5) \times Z_{2} \times Z_{2} \times U(1)
$$

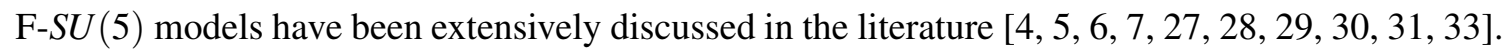

\section{Mordell-Weil $U(1)$ 's and discrete symmetries}

In the previous section we presented the classification of the non-abelian singularities of the elliptic fiber, subject to restrictions arising from Kodaira classification. Adopting the interpretation that these correspond to non-abelian gauge symmetries, we were able to determine the GUT gauge group of the potential F-theory models. There is considerable activity [56]-[ [75] on the role of the abelian sector related to the rational sections of the elliptic curves. Subsequently, we focus in some related issues.

In the introductory section we have seen that there is a class of abelian symmetries, associated to rational sections of the elliptic curves. Since the internal space is elliptically fibred, these $U(1)$ 's may manifest themselves in potential low energy effective models. From analyses of phenomenological models, we know that such symmetries are extremely useful in order to prevent unwanted terms in the lagrangian. It seems that such abelian symmetries are indispensable when constructing an F-theory effective model, however, a Kodaira-type classification is lacking up to now. From the Mordell-Weil theorem we only know that these are related to the rational sections defined on elliptic curves but the rank of this group is not known. The Mordell-Weil group can be written as

$$
\mathscr{E}(\mathscr{K})=\underbrace{Z \oplus Z \oplus \cdots \oplus Z}_{n} \oplus \mathscr{G} \equiv Z^{n} \oplus \mathscr{G}
$$


Here $n$ is the rank of the Abelian group, while $\mathscr{G}$ is the torsion subgroup and $\mathscr{K}$ is the number field. According to a theorem by Mazur [ㅈ] (see also [[Z]), the possible torsion subgroups are either $\mathbf{Z}_{k}, k=1,2, \ldots, 10,12$ or the direct $\operatorname{sum} \mathbf{Z}_{2} \oplus \mathbf{Z}_{2 k}$ with $k=1,2,3,4$.

$$
\mathscr{G}= \begin{cases}\mathbf{Z}_{n} & n=1,2, \ldots, 10,12 \\ \mathbf{Z}_{2 k} \oplus \mathbf{Z}_{2} & k=1,2,3,4\end{cases}
$$

A specific choice of the coefficients in an elliptic curve equation eventually will fix the symmetries of the effective GUT model. For a simple demonstration on the appearance of such symmetries, let us see how a $\mathbf{Z}_{2}$ discrete symmetry can arise [[Z8]. Under a $\mathbf{Z}_{2}$ action, a point $P$ on an elliptic curve is identified with its opposite, $-P$. From the group law, (see figure $\square) P+(-P)=O$, hence $P+P=O$, where for the Weierstraß form the zero element $O$ is taken to infinity. This implies that line $O P$ must be tangent to $P$. If we put $P$ at the origin, $(0,0)$ then $d y / d x=\infty$. For example, the elliptic curve $y^{2}=x\left(x^{2}+x+1\right)$ has a $\mathbf{Z}_{2}$ symmetry at the origin $(0,0)$.

\subsection{On GUT Models with Mordell-Weil $U(1)$ 's}

In the geometric picture of F-theory discussed previously, the elliptic fibration assumed over a base $B_{3}$ can be defined as a holomorphic section of the fourfold. In the following, the possibility of having a fibration of an elliptic curve with two rational sections, including the zero (universal) section will be examined. This leads to a rank-one Mordell-Weil group, or a theory with one $U(1)$ symmetry in addition to the non-abelian GUT group. From the phenomenological point of view this is one of the most viable possibilities. GUT models with an additional abelian factor and perhaps a discrete symmetry arising from the torsion part (6. لل. $)$, are probably adequate to impose sufficient constraints on superpotential terms.

However, in general, it is not easy to identify the $U(1)$ symmetries by starting directly form the Weierstraß form. Instead, it is more feasible to start with a different representation of the elliptic curve where the Mordell-Weil rank and other characteristics are more transparent. Once we have identified the abelian structure, in order to study the non-abelian group we convert our equation to the ordinary Weierstraß form using the appropriate birational transformation. To derive the
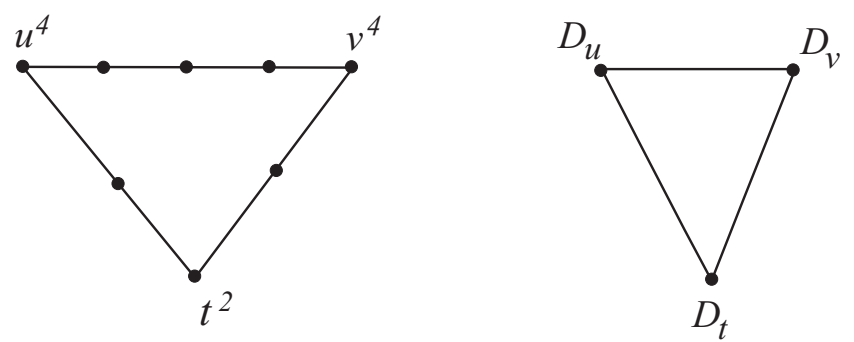

Figure 8: The reflexible polygon for the model $\mathbf{P}_{(1,1,2)}$ with one lattice point in its interior in accordance with the genus-one elliptic curve.

equation of such a hypersurface, following the analysis of [56], we start with a point $P$ associated to the holomorphic (zero) section and a rational point $Q$ on an elliptic curve. We introduce the degree-two line bundle $\mathscr{M}=\mathscr{O}(P+Q)$ and denote $u$ and $v$ its two independent sections with weights $[1: 1]$ generating the group $H^{0}(\mathscr{M})$. The space $H^{0}(2 \mathscr{M})$ should have four independent 
sections. Given $u$ and $v$ we are able to generate only three, namely, $u^{2}, v^{2}$ and $u v$. Thus we need to introduce a new one -let us call this $t$ - with weight 2 , so we are in a $\mathbf{P}_{(1,1,2)}$ projective space of three sections $[u, v, t]$ with weights $[1: 1: 2]$ respectively. From $u, v, t$ we can form six sections of degree 6 (namely $u^{3}, v^{3}, u v^{2}, u^{2} v$ and $t u, t v$ ) which match exactly the number of independent sections of $H^{0}(3 \mathscr{M})$. But $u, v, t$ generate nine sections for $H^{0}(4 \mathscr{M})$ exceeding the independent ones by one. Hence there has to be a constraint among them which defines a hyper-surface in the weighted projective space $\mathbf{P}_{(1,1,2)}$ given by the equation of the form

$$
t^{2}+a_{0} u^{2} t+a_{1} u v t+a_{2} v^{2} t=b_{0} u^{4}+b_{1} u^{3} v+b_{2} u^{2} v^{2}+b_{3} u v^{3}+b_{4} v^{4}
$$

where $b_{i}, a_{j}$ are coefficients in the specific field $\mathscr{K}$ we are interested in. The $\mathbf{P}_{(1,1,2)}$ projective

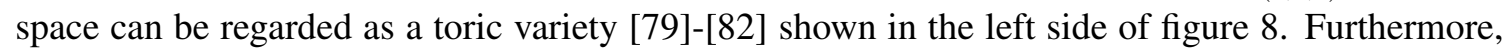
we can identify three divisors. For $t=v=0, u \neq 0$ the divisor $D_{u}=[1: 0: 0]$, for $t=u=0, v \neq 0$ the divisor $D_{v}=[0: 1: 0]$, and for $u=v=0, t \neq 0$, the divisor $D_{t}=[0: 0: 1]$. These are indicated on the right side of the same figure. Without loss of generality [56] in order to avoid complications with square roots etc, we can simplify this equation to:

$$
t^{2}+a_{2} v^{2} t=u\left(b_{0} u^{3}+b_{1} u^{2} v+b_{2} u v^{2}+b_{3} v^{3}\right)
$$

Having constructed the elliptic curve equation with one Mordell-Weil $U(1)$, we would like now to transform this equation to the familiar $\mathbf{P}_{(2,3,1)}$ model. In fact this is inevitable; in order to identify the non-abelian part of the gauge symmetry, we need to read off the singularity structure from the coefficients in the Weierstraß form. It can be proved that the conversion can occur by two sets of equations [ㅁ] relating the sections of the $\mathbf{P}_{(1,1,2)}$ model to those of $\mathbf{P}_{(2,3,1)}$. Both transformations lead to equivalent results. The simplest one is [प] $]$ :

$$
\begin{aligned}
v & =\frac{a_{2} y}{b_{3}^{2} u^{2}-a_{2}^{2}\left(b_{2} u^{2}+x\right)} \\
t & =\frac{b_{3} u y}{b_{3}^{2} u^{2}-a_{2}^{2}\left(b_{2} u^{2}+x\right)}-\frac{x}{a_{2}} \\
u & =z
\end{aligned}
$$

We substitute the above to $\mathbf{P}_{(1,1,2)}$ model and we recover the Tate's form

$$
\begin{aligned}
y^{2}+2 \frac{b_{3}}{a_{2}} x y z \pm b_{1} a_{2} y z^{3}= & x^{3} \pm\left(b_{2}-\frac{b_{3}^{2}}{a_{2}^{2}}\right) x^{2} z^{2} \\
& -b_{0} a_{2}^{2} x z^{4}-b_{0} a_{2}^{2}\left(b_{2}-\frac{b_{3}^{2}}{a_{2}^{2}}\right) z^{6}
\end{aligned}
$$

This is indeed in the desired $\mathbf{P}_{(2,3,1)}$ form however not all of the Tate's coefficients are independent. Comparing with the standard Tate's form given in (4.]) we observe the relations

$$
\begin{aligned}
& \alpha_{1}= \pm 2 \frac{b_{3}}{a_{2}} \\
& \alpha_{2}=b_{2}-\frac{b_{3}^{2}}{a_{2}^{2}}
\end{aligned}
$$




$$
\begin{aligned}
& \alpha_{3}= \pm b_{1} a_{2} \\
& \alpha_{4}=-b_{0} a_{2}^{2} \\
& \alpha_{6}=-\left(b_{2}-\frac{b_{3}^{2}}{a_{2}^{2}}\right) b_{0} a_{2}^{2}
\end{aligned}
$$

In particular, notice that the following relation holds

$$
\alpha_{6}=\alpha_{2} \alpha_{4}
$$

As we shall see in the following, this relation inevitably implies constraints on the non-abelian singularities. We restrict here the analysis in the Tate's form of the Weierstraß equation since it is this form that we automatically obtain from the birational map. Hence, we assume the local expansion of the Tate's coefficients which as a function of the "normal" coordinate they are given by (5.1) and (5.2).

To see the implications of the relation $\alpha_{6}=\alpha_{4} \alpha_{2}$, we need to substitute in it the specific types of coefficients for each non-abelian group shown in Table $\square$.

We start the investigation with the $S U(n)$ singularities. According to Table $\square$ we must treat separately even $S U(2 m)$ and odd $S U(2 m+1)$ cases.

1. $S U(2 m)$ case. The vanishing orders of $\alpha_{k}$ 's for $S U(2 n)$ groups are

$$
\alpha_{2}=\alpha_{2,1} w, \alpha_{4}=\alpha_{4, m} w^{m}, \alpha_{6}=\alpha_{6,2 m} w^{2 m}
$$

Substitution into (6.8) gives

$$
\alpha_{2,1} \alpha_{4, m} w^{m+1}=\alpha_{6,2 m} w^{2 m}
$$

which is satisfied only for $m=1$, implying that only $S U(2)$ is compatible.

2. $S U(2 m+1)$ case. Reading off the minimal powers of $\alpha_{k}$ 's from Table $\square$, we get

$$
\alpha_{2,1} \alpha_{4, m} w^{m+2}=\alpha_{6,2 m} w^{2 m+1}
$$

This is also valid for $m=1$, hence only $S U(3)$ is admissible.

Extending this analysis to the rest of the entries in Tate's table, one finds that the most interesting cases arise for the exceptional groups. We observe that under the particular birational map to Tate's form the only non-trivial admissible non-abelian singularities are $\mathscr{E}_{6}$ and $\mathscr{E}_{7}$.

For $\mathscr{E}_{6}$ in particular we have

$$
\alpha_{2} \alpha_{4} \propto \alpha_{2,2} \alpha_{4,3} w^{5}, \alpha_{6} \propto \alpha_{6,5} w^{5}
$$

i.e, the $w$ powers match and therefore we only need to impose the equality constraint

$$
\alpha_{2,2} \alpha_{4,3}=\alpha_{6,5}
$$




\begin{tabular}{|c|c|c|c|c|c|c|c|}
\hline Type & Group & $a_{1}$ & $a_{2}$ & $a_{3}$ & $a_{4}$ & $a_{6}$ & $\Delta$ \\
\hline$I_{0}$ & - & 0 & 0 & 0 & 0 & 0 & 0 \\
\hline$I_{1}$ & - & 0 & 0 & 1 & 1 & 1 & 1 \\
\hline$I_{2}^{s}$ & $S U(2)$ & 0 & 1 & 1 & 1 & 2 & 2 \\
\hline$I_{3}^{s}$ & $S U(3)$ & 0 & 1 & 1 & 2 & 3 & 3 \\
\hline$I_{1}^{* n s}$ & $S O(9)$ & 1 & 1 & 2 & 3 & 4 & 7 \\
\hline$I_{0}^{* n}$ & $S O(8)^{*}$ & 1 & 1 & 2 & 2 & 3 & 6 \\
\hline$I V^{* s}$ & $\mathscr{E}_{6}$ & 1 & 2 & 2 & 3 & 5 & 8 \\
\hline$I I I^{* S}$ & $\mathscr{E}_{7}$ & 1 & 2 & 3 & 3 & 5 & 9 \\
\hline
\end{tabular}

Table 3: Selected cases of Tate's coefficients satisfying the relation $\alpha_{6}=\alpha_{2} \alpha_{4}$. The Standard Model is naturally embedded in the exceptional groups only.

\begin{tabular}{|cccccc|}
\hline Group & $a_{2}$ & $b_{0}$ & $b_{1}$ & $b_{2}$ & $b_{3}$ \\
\hline $\mathscr{E}_{6}$ & 1 & 1 & 1 & 2 & 2 \\
& 0 & 3 & 2 & 2 & 1 \\
\hline $\mathscr{E}_{7}$ & 1 & 1 & 2 & 2 & 2 \\
& 0 & 3 & 3 & 2 & 1 \\
\hline
\end{tabular}

Table 4: The vanishing order of the coefficients $b_{k} \sim b_{k, n} w^{n}$, the two exceptional symmetry models.

Once this condition is satisfied, we also need to check the remaining coefficients constrained by equations (6.7). To investigate these relations, we express all coefficients in terms of $a_{2}$. Assuming that the latter is given in terms of an unspecified power of the coordinate,

$$
a_{2} \propto w^{n}
$$

we find that a consistent solution exists in accordance with

$$
b_{0}=-\alpha_{43} w^{3-2 n}, b_{1}=\alpha_{32} w^{2-n}, b_{2}=\left(\alpha_{22}+\alpha_{11}^{2} / 2\right) w^{2}, b_{3}=\left(\alpha_{11} / 2\right) w^{n+1}
$$

Requiring the $b_{0}$ coefficient to be a positive power in $w$ we see that this leaves two possibilities for the integer $n$, namely $n=0,1$.

Substituting (6.9) into the equations (6.7) we find

$$
\alpha_{1}=\alpha_{11} w, \alpha_{2}=\alpha_{2} w^{2}, \alpha_{3}=\alpha_{32} w^{2}, \alpha_{4}=\alpha_{43} w^{3}, \alpha_{6}=\alpha_{65} w^{5}
$$

As can be checked in Table $\mathbb{\Psi}$ this is just the requirement to obtain an $\mathscr{E}_{6}$ singularity. We compute the discriminant to find

$$
\Delta=-27 \alpha_{32}^{4} w^{8}+\mathscr{O}\left(w^{9}\right)
$$

which, as expected has vanishing order 8 .

- Repeating the analysis of the $\mathscr{E}_{7}$ case, we end up with the conditions on $b_{i}$ 's listed in the

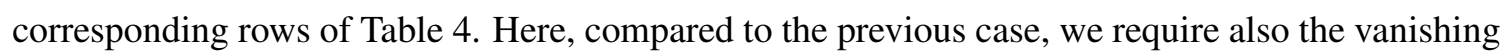


of the coefficient $\alpha_{32}$ so that $\alpha_{3}=\alpha_{3,3} w^{3}$. It is also straightforward to see that $\Delta \propto w^{9}$ in accordance with Table $\square$. Finally, notice that for the $\mathscr{E}_{8}$ case, the condition $a_{2} a_{4}=a_{6}$ cannot be fulfilled.

From the above analysis, we have seen that in the presence of an additional rational section which is associated to an extra $U(1)$ symmetry -as long as the minimal requirements on $\alpha_{n}$ of Table $\square$ are implemented-, the available non-abelian groups compatible with the restrictions are $S U(3), S U(2)$ and the $\mathscr{E}_{6}$ and $\mathscr{E}_{7}$. From these, only the exceptional groups are adequate to include the complete gauge symmetry of the SM. The $\mathscr{E} 6$ model looks the most promising and has been extensively analysed in the literature. A detailed analysis can be found in [B]].

\section{Conclusions}

In this lecture, a few basic tools for F-theory model building have been described. Current F-theory constructions are based on the elliptically fibred internal space with $\mathscr{E} 8$ being the highest admissible singularity. We started our investigation by presenting a few necessary ingredients, namely some elementary notions related to elliptic curves, elliptic fibration through the Weierstraß model and F-theory basics. Subsequently, we introduced the main tools in order to build viable models.

We discussed a class of F-theory effective models which are fully embedded in $\mathscr{E}$. As a consequence, the GUT symmetry is a subgroup of $\mathscr{E}_{8}$ while the commutant incorporates any additional symmetry of discrete or continuous nature which could be used to put constraints on the effective lagrangian.

Next, we analysed abelian and discrete symmetries emerging from the a non-trivial MordellWeil group, i.e. the group of rational points of the elliptic curves associated to the fibration. In this approach, one constructs a representation of the elliptic curve with the desired rational sections and then finds the birationally equivalent Weierstraß equation.

In the presence of one Mordell-Weil factor in particular, we have shown that the birational transformations to Tate's form gives two viable gauge symmetries which are $\mathscr{E}_{6} \times U(1)_{M W}$ or $\mathscr{E}_{7} \times U(1)_{M W}$. Although in the presence of a Mordell-Weil $U(1)$ the non-abelian sector seems to be constrained, yet these exceptional groups contain all the well known GUTs, such as $S U(5)$, $S O(10)$ and the like, which can be readily obtained once we break the initial symmetry by a suitable mechanism, such as flux breaking, Wilson lines mechanism etc. The rather interesting fact in the procedure, is that the $U(1)_{M W}$ symmetry is not necessarily identified with some generator of the Cartan subalgebra of $\mathscr{E}_{8}$. This means that the $U(1)_{M W}$ charges of the non-abelian representations are not necessarily the usual ones. Furthermore, the torsion group has a rich structure of discrete symmetries which can also be symmetries of the effective lagrangian. Perhaps issues such as proton stability, the $\mu$-term and flavour physics find their solutions in a judicious choice of these symmetries.

\section{References}

[1] C. Vafa, Nucl. Phys. B 469 (1996) 403 [arXiv:hep-th/9602022].

[2] J. J. Heckman and C. Vafa, Nucl. Phys. B 837 (2010) 137 [arXiv:0811.2417].

[3] R. Donagi and M. Wijnholt, arXiv:0802.2969. 
[4] C. Beasley, J. J. Heckman and C. Vafa, JHEP 0901 (2009) 058 [arXiv:0802.3391].

[5] R. Donagi and M. Wijnholt, arXiv:0808.2223.

[6] C. Beasley, J. J. Heckman and C. Vafa, JHEP 0901 (2009) 059 [arXiv:0806.0102 ].

[7] R. Blumenhagen, T. W. Grimm, B. Jurke and T. Weigand, Nucl. Phys. B 829 (2010) 325 [arXiv:0908.1784].

[8] A. Font and L. E. Ibanez, JHEP 0902 (2009) 016 [arXiv:0811.2157 ].

[9] A. Font, F. Marchesano, D. Regalado and G. Zoccarato, JHEP 1311 (2013) 125 [arXiv:1307.8089 [hep-th]].

[10] L. B. Anderson, J. J. Heckman and S. Katz, JHEP 1405 (2014) 080 [arXiv:1310.1931 [hep-th]].

[11] F. Marchesano, D. Regalado and G. Zoccarato, arXiv:1503.02683 [hep-th].

[12] H. Hayashi, T. Kawano, R. Tatar and T. Watari, Nucl. Phys. B 823 (2009) 47 [arXiv:0901.4941 ].

[13] S. Cecotti, M. C. N. Cheng, J. J. Heckman and C. Vafa, arXiv:0910.0477 [hep-th].

[14] J. J. Heckman, A. Tavanfar and C. Vafa, JHEP 1008 (2010) 040 [arXiv:0906.0581 [hep-th]].

[15] G. K. Leontaris and N. D. Tracas, Eur. Phys. J. C 67 (2010) 489 [arXiv:0912.1557 [hep-ph]].

[16] G. K. Leontaris and G. G. Ross, JHEP 1102 (2011) 108 [arXiv:1009.6000 ].

[17] L. Aparicio, A. Font, L. E. Ibanez and F. Marchesano, JHEP 1108 (2011) 152 [arXiv:1104.2609 ].

[18] P. G. Camara, E. Dudas and E. Palti, JHEP 1112 (2011) 112 [arXiv:1110.2206].

[19] E. Palti, JHEP 1207 (2012) 065 [arXiv:1203.4490].

[20] A. Font, L. E. Ibanez, F. Marchesano and D. Regalado, JHEP 1303 (2013) 140 [Erratum-ibid. 1307 (2013) 036] [arXiv:1211.6529].

[21] A. Font, F. Marchesano, D. Regalado and G. Zoccarato, JHEP 1311 (2013) 125 [arXiv:1307.8089 ].

[22] G. K. Leontaris and N. D. Vlachos, Phys. Lett. B 704 (2011) 620 [arXiv:1105.1858 [hep-th]].

[23] J. J. Heckman, Ann. Rev. Nucl. Part. Sci. 60 (2010) 237 [arXiv:1001.0577].

[24] T. Weigand, Class. Quant. Grav. 27 (2010) 214004 [arXiv:1009.3497].

[25] G. K. Leontaris, PoS CORFU 2011 (2011) 095 [arXiv:1203.6277].

[26] A. Maharana and E. Palti, Int. J. Mod. Phys. A 28 (2013) 1330005 [arXiv:1212.0555].

[27] E. Dudas and E. Palti, JHEP 1001 (2010) 127 [arXiv:0912.0853].

[28] T. W. Grimm and T. Weigand, Phys. Rev. D 82 (2010) 086009 [arXiv:1006.0226].

[29] A. P. Braun, A. Collinucci and R. Valandro, Nucl. Phys. B 856 (2012) 129.

[30] S. F. King, G. K. Leontaris and G. G. Ross, Nucl. Phys. B 838 (2010) 119 [arXiv:1005.1025].

[31] J. C. Callaghan, S. F. King, G. K. Leontaris and G. G. Ross, JHEP 1204 (2012) 094 [arXiv:1109.1399]

[32] J. C. Callaghan and S. F. King, JHEP 1304 (2013) 034 [arXiv:1210.6913].

[33] J. Marsano, H. Clemens, T. Pantev, S. Raby and H. H. Tseng, JHEP 1301 (2013) 150 [arXiv:1206.6132 [hep-th]].

[34] I. Antoniadis and G. K. Leontaris, JHEP 1208 (2012) 001 [arXiv:1205.6930 [hep-th]]. 
[35] I. Antoniadis and G. K. Leontaris, Eur. Phys. J. C 73 (2013) 2670 [arXiv:1308.1581]

[36] L. E. Ibanez, A. N. Schellekens and A. M. Uranga, Nucl. Phys. B 865 (2012) 509 [arXiv:1205.5364].

[37] M. Berasaluce-Gonzalez, P. G. Camara, F. Marchesano, D. Regalado and A. M. Uranga, JHEP 1209, 059 (2012) [arXiv:1206.2383].

[38] F. Marchesano, D. Regalado and L. Vazquez-Mercado, JHEP 1309 (2013) 028 [arXiv:1306.1284].

[39] P. Anastasopoulos, M. Cvetic, R. Richter and P. K. S. Vaudrevange, JHEP 1303 (2013) 011 [arXiv:1211.1017].

[40] G. K. Leontaris, arXiv:1501.06499 [hep-th].

[41] M. Cveti, R. Donagi, D. Klevers, H. Piragua and M. Poretschkin, arXiv:1502.06953 [hep-th].

[42] P. Anastasopoulos, R. Richter and A. N. Schellekens, arXiv:1502.02686 [hep-th].

[43] G. Honecker and W. Staessens, JHEP 1310 (2013) 146 [arXiv:1303.4415].

[44] A. Karozas, S. F. King, G. K. Leontaris and A. Meadowcroft, JHEP 1409 (2014) 107 [arXiv:1406.6290].

[45] C. Mayrhofer, E. Palti, O. Till and T. Weigand, arXiv:1410.7814].

[46] Joseph H. Silverman and John T. Tate, Rational Points on Elliptic Curves ? November 18, 1994

[47] J. H. Silverman, The arithmetic of elliptic curves, - Springer, 2nd ed. 2009.

[48] J. Cassels, Lectures on Elliptic Curves, Cambridge, 1991.

[49] F. Denef, arXiv:0803.1194.

[50] A. Sen, Nucl. Phys. B 475 (1996) 562 [hep-th/9605150].

[51] K. Kodaira, “On Compact Analytic Surfaces II,” Annals of Math. 77, 563-626 (1963)

[52] M. Bershadsky, K. A. Intriligator, S. Kachru, D. R. Morrison, V. Sadov and C. Vafa, Nucl. Phys. B 481 (1996) 215 [arXiv:hep-th/9605200].

[53] D. R. Morrison and C. Vafa, Nucl. Phys. B 476 (1996) 437 [hep-th/9603161].

[54] M. Esole and S. T. Yau, Adv. Theor. Math. Phys. 17 (2013) 1195 [arXiv:1107.0733].

[55] J. Tate, "Algorithm for Determining the Type of a Singular Fiber in an Elliptic Pencil," in Modular Functions of One Variable IV, Lecture Notes in Math. vol. 476, Springer-Verlag, Berlin (1975).

[56] D. R. Morrison and D. S. Park, JHEP 1210 (2012) 128 [arXiv:1208.2695].

[57] M. Cvetic, T. W. Grimm and D. Klevers, JHEP 1302 (2013) 101 [arXiv:1210.6034]

[58] C. Mayrhofer, E. Palti and T. Weigand, JHEP 1303 (2013) 098 [arXiv:1211.6742]

[59] V. Braun, T. W. Grimm and J. Keitel, JHEP 1309 (2013) 154 [arXiv:1302.1854 ]

[60] J. Borchmann, C. Mayrhofer, E. Palti and T. Weigand, Phys. Rev. D 88 (2013) 4, 046005 [arXiv:1303.5054].

[61] M. Cvetic, D. Klevers and H. Piragua, JHEP 1306 (2013) 067 [arXiv:1303.6970]

[62] T. W. Grimm, A. Kapfer and J. Keitel, JHEP 1307 (2013) 115 [arXiv:1305.1929]

[63] V. Braun, T. W. Grimm and J. Keitel, JHEP 1312 (2013) 069 [arXiv:1306.0577]

[64] M. Cvetic, A. Grassi, D. Klevers and H. Piragua, JHEP 1404 (2014) 010 [arXiv:1306.3987] 
[65] J. Borchmann, C. Mayrhofer, E. Palti and T. Weigand, Nucl. Phys. B 882 (2014) 1 [arXiv:1307.2902]

[66] M. Cvetic, D. Klevers and H. Piragua, JHEP 1312 (2013) 056 [arXiv:1307.6425].

[67] M. Cvetic, D. Klevers, H. Piragua and P. Song, JHEP 1403 (2014) 021 [ arXiv:1310.0463].

[68] V. Braun and D. R. Morrison, JHEP 1408 (2014) 132 [arXiv:1401.7844]

[69] S. Krippendorf, D. K. Mayorga Pena, P. K. Oehlmann and F. Ruehle, JHEP 1407 (2014) 013 [arXiv:1401.5084]

[70] I. Antoniadis and G. K. Leontaris, Phys. Lett. B 735 (2014) 226 [arXiv:1404.6720]

[71] M. Del Zotto, J. J. Heckman, D. R. Morrison and D. S. Park, arXiv:1412.6526

[72] I. García-Etxebarria, T. W. Grimm and J. Keitel, JHEP 1411 (2014) 125 [arXiv:1408.6448].

[73] D. Klevers, D. K. Mayorga Pena, P. K. Oehlmann, H. Piragua and J. Reuter, [arXiv:1408.4808].

[74] C. Mayrhofer, E. Palti, O. Till and T. Weigand, JHEP 1412 (2014) 068 [arXiv:1408.6831].

[75] M. Esole, M. J. Kang and S. T. Yau, arXiv:1410.0003 [hep-th].

[76] Mazur, B, "Modular curves and the Eisenstein Ideal" Publications Mathématiques de l' I.H.É. S., tome 47(1977), p. 33-186

[77] D.S. Kubert, Universal bounds on the torsion of elliptic curves, in Proc. London Math. Soc.(3) 33 (1976)193.

[78] P. S. Aspinwall and D. R. Morrison, JHEP 9807 (1998) 012 [hep-th/9805206].

[79] M. Kreuzer and H. Skarke, Commun. Math. Phys. 185 (1997) 495 [hep-th/9512204].

[80] P. Candelas and A. Font, Nucl. Phys. B 511 (1998) 295 [hep-th/9603170].

[81] V. Bouchard and H. Skarke, Adv. Theor. Math. Phys. 7 (2003) 205 [hep-th/0303218].

[82] A. Grassi and V. Perduca, Adv. Theor. Math. Phys. 17 (2013) 741 [arXiv:1201.0930]. 